\title{
A Mobile System for Assessment of Physiological Response to Posture Transitions
}

\author{
Emil Jovanov, Senior Member, IEEE, Mladen Milosevic, Member, IEEE, \\ Aleksandar Milenković, Senior Member, IEEE, \\ University of Alabama in Huntsville, Huntsville, Alabama, U.S.A
}

\begin{abstract}
Posture changes initiate a dynamic physiological response that can be used as an indicator of the overall health status. We introduce an inconspicuous mobile wellness monitoring system (imWell) that continuously assesses the dynamic physiological response to posture transitions during activities of daily living. We use Zephyr BioHarness 3 physiological monitor that continually reports heart activity and physical activity via Bluetooth to a personal device. The personal device processes all the data in real-time, recognizes posture transitions from accelerometer data, characterizes dynamic heart response to posture changes, annotates, logs, and uploads heart activity data to our mHealth server. In this paper we present algorithms for detection of posture transitions and heart activity characterization during a sit-to-stand transition. The proposed system was tested on seven healthy subjects performing a predefined protocol. The total average and standard deviation for sit-to-stand transition time is $2.7 \pm 0.69 \mathrm{~s}$, resulting in the change of heart rate of $27.36 \pm 9.30 \mathrm{bpm}$ (from $63.3 \pm 9.02 \mathrm{bpm}$ to $90.66 \pm 10.09 \mathrm{bpm})$.
\end{abstract}

\section{INTRODUCTION}

Cardiovascular diseases represent the leading cause of death in the United States. The current practices in monitoring health status are expensive, often insufficient, and limited to monitoring in healthcare facilities. Patients usually consult cardiologists only after experiencing first symptoms of deteriorating cardiac status. Cardiologists usually administer exercise stress test to assess cardiac status. Standard stress tests are bike or treadmill exercise supervised by a doctor or a trained technician to determine the level of exercise a patient can tolerate. The tests are usually followed by prescribed therapeutic lifestyle changes including modification of diet and development of an exercise program with a follow-up after several months. However, the clinicians do not have tools to assess the patients' progress and their compliance to the prescribed therapies. Patients and healthy users lack tools to monitor their own cardiac status and to manage healthy lifestyle.

This work was supported in part by the University of Alabama in Huntsville under Grant UAH234343 and NSF CNS-1205439.

E. Jovanov is with the Electrical and Engineering Department, University of Alabama in Huntsville, Huntsville, AL 35899 (Phone: 256824-5094; fax: 256-824-6803; e-mail: emil.jovanov@uah.edu).

M. Milosevic and A. Milenkovic are with the Electrical and Computer Engineering Department, University of Alabama in Huntsville, Huntsville, AL 35899 (e-mail: mladen.milosevic@uah.edu and milenka@uah.edu).
Emergence and proliferation of lightweight and wearable physiological sensors enable the design of digital assistants that are capable of continual sensing and processing of cardiac activity and physical activity. A number of new consumer-grade devices for health and wellness monitoring has been introduced [1], [2]. Examples include pedometers that count number of steps and calories, and more sophisticated devices, such as heart and metabolic monitors. On the other side, an increasing number of users actively monitor their own health and fitness status [3]. The availability of affordable wearable devices and their consumer acceptance create new opportunities for individual users and healthcare professionals.

Posture changes, such as transitions from sitting to standing, induce a physiological response that can be used to characterize fitness status of the user. Figure 1(b) shows heart rate recorded during a posture transition from sitting to standing for a healthy subject (blue line) and a subject with cardiac condition (red line) The heart rate in the healthy subject quickly increases as a response to the physical activity and then returns to a level that corresponds to the new homeostasis.

We hypothesize that characterizing this dynamic response, including a change in the heart rate and time to reach the maximum, can be used to indicate the subject's cardiac status and overall wellness. Long term monitoring of cardiac health and physical activity during activities of daily living can provide indication of short term and long term changes of cardiac status and fitness. The goal of this project is to develop an inconspicuous wearable cardiac and wellness assistant called imWell (inconspicuous mobile Wellness) for efficient unobtrusive monitoring of physical activity, cardiac health, and overall physical wellness.

The imWell system continually records interbeat intervals and processes accelerometer data to recognize posture transitions (Section III.A). For detected sit-to-stand posture transitions the system characterizes dynamic heart response by extracting transition timestamp, duration, heart rate before and after transition, and the time needed to reach the maximum heart rate from the beginning of the transition (Section 0). This way we treat each sit-to-stand posture change, which happens dozens of times per day, as a mini "exercise test". Analyzing the results of these tests over long period of time, could be used to assess cardiac status and fitness. 

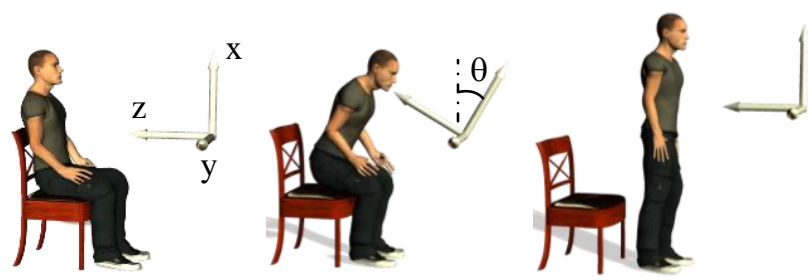

(a)

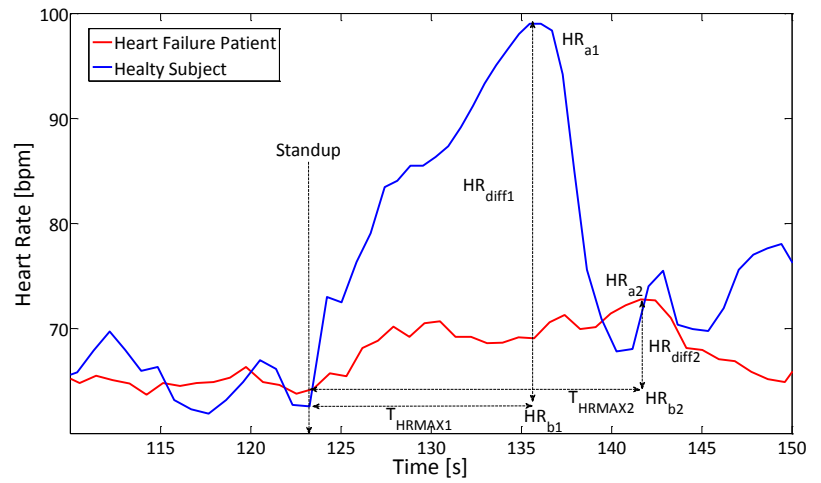

(b)

Figure 1. Modeling change of heart activity induced by transition from sitting to standing

Similar to a personal digital assistant that helps individuals manage day-to-day data needs, the $\mathrm{imWell}$ system serves as a personal assistant targeted to help individuals manage their cardiac health and physical wellness. Such a system can benefit: (a) healthy subjects, allowing them to track and manage the overall cardiac health status and physical fitness over long periods of time, (b) cardiac rehabilitation patients, allowing them to monitor and guide their rehabilitation and detect early deterioration of cardiac status; and (c) physicians and healthcare professional, allowing them to monitor patient's cardiac health status and to verify their compliance to prescribed lifestyle changes and exercises.

\section{RELATED WORK}

Recognition and quantification of human activities using small wearable sensors during activities of daily living has been increasingly used in many applications. Automatic activity recognition and quantification systems that utilize inertial sensors are proposed for long-term health and fitness monitoring [4], [5], assessing of mobility in elderly and people with Parkinson's disease [6]-[8], automatic fall detection [9], [10], and rehabilitation [11]. Approaches for automatic activity recognition used by researchers vary in number, type, and placement of utilized sensors, as well as in processing of recorded signals. While some researchers used multiple sensors for automatic activity recognition [12]-[14] increasing number of projects use a single inertial sensor [15]-[18] usually placed on the chest.

One of the most challenging tasks in automatic activity recognition is detection and differentiation of posture transitions sit-to-stand and stand-to-sit. A number of researchers monitored angle of trunk tilt to detect possible posture changes by detecting situations when the angle exceeds certain threshold [15], [17]-[19]. While similar approaches for detection of possible posture transition are used, researchers used significantly different approaches in order to differentiate aforementioned transitions. Godfrey et al. [18] used vertical velocity, Fleury et al. [19] used wavelet-based pattern recognition on accelerometer data and tilt angle, while Fuentes at al. [17] used angle and forward and vertical acceleration.

\section{METHODS}

Our system relies on physiological monitor Zephyr BioHarness 3 to capture both inertial and physiological data (acceleration and RR-intervals). The monitor is placed in a chest belt worn by the user and communicates the data via Bluetooth to a personal device. The personal device (a) processes the signals to detect and timestamp transitions using data from inertial sensors, (b) extracts, characterizes, and records dynamic heart response to sit-to-stand transitions, and (c) uploads the records to a mHealth server [20].

\section{A. Posture Transition Detection}

Figure 1(a) shows a user during a transition from seating to standing and orientation of the Zephyr's inertial sensor, with vertical axis (x), lateral (y), and sagittal (z). The Zephyr monitor is mounted in side strap located under subject's left arm. The Zephyr's inertial sensor samples and reports acceleration components $A_{x}, A_{y}$, and $A_{z}$ with the sampling frequency of $50 \mathrm{~Hz}$. When the user is standing still or seating in the upright position we should observe $A_{x}=-1 g, A_{y}=A_{z}=0$, where the $\mathrm{g}$ is the Earth gravitation $\left(9.81 \mathrm{~m} / \mathrm{s}^{2}\right)$.

The first step in identifying possible posture transitions is to detect a change in the upper body angle relative to the upright position, $\theta$, and a change in the acceleration vector magnitude, AccMag. The upper body angle is calculated as follows: $\theta=\arctan \left(\mathrm{A}_{\mathrm{z}} / \mathrm{A}_{\mathrm{x}}\right)$, where $\theta=0$ degrees in the upright position as shown in Figure 1(a). It has a characteristic signature during posture transitions sit-to-stand and stand-to-sit. Figure 3(b) shows the angle during a sit-to-stand transition. We can locate 3 characteristic points in the angle signature - two purple circles mark the beginning (BeginTransition) and the end of the transition (EndTransition), and purple diamond marks the angle peak (AnglePeak).

To identify a possible posture transition we search for a segment where the angle rises above a certain threshold. However, the angle $\theta$ may include offsets caused by imperfect monitor placement and monitor's movements relative to the body. To eliminate the impact of the offsets we calculate the standard deviation of the angle $\theta$ on a sliding window of 1 second, $\operatorname{stdev}(\theta)$, and use it to locate possible posture transitions. This signal is searched for maximums in segments that are above a certain threshold. Once we locate candidate segments we search for three characteristic points in the original angle (BeginTransition, AnglePeak, EndTransition).

To estimate the level of physical activity we calculate the standard deviation of the acceleration vector magnitude on a sliding window of 1 second, stdev(AccMag). The 


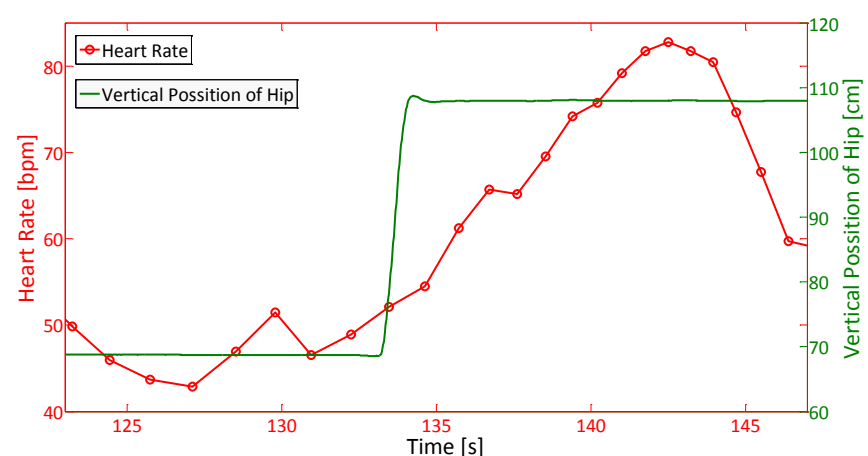

(a)

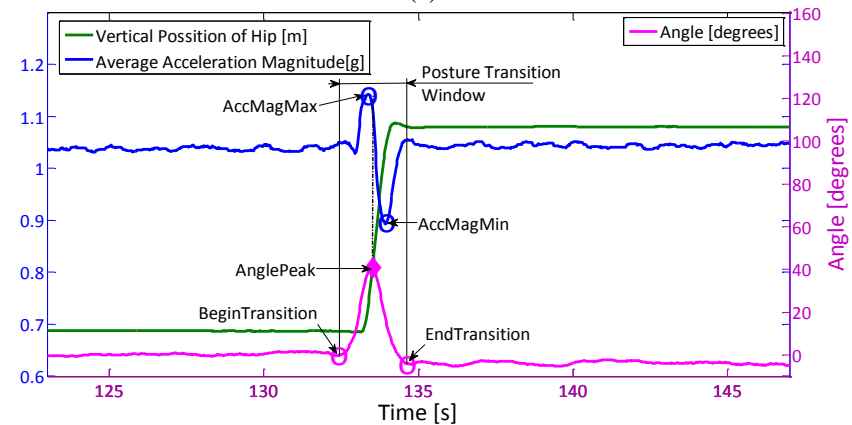

(b)

Figure 3. An example of physiological response during transition from sitting to standing: a) real-time heart rate calculated from RR intervals and hip position during transition; b) angle and magnitude calculated from accelerometer signals and hip position during transition.

stdev(AccMag) is used to distinguish between posture transitions and motion artifacts such as leaning forward/backward/sideways in a chair. If the maximum stdev(AccMag) in a candidate segment is higher than a certain threshold, we mark this segment as a true transition. In true transition segments, we search the acceleration vector magnitude for two characteristic points, marked by blue circles in Figure 2(b), representing the maximum and the minimum (AccMagMax, AccMagMin).

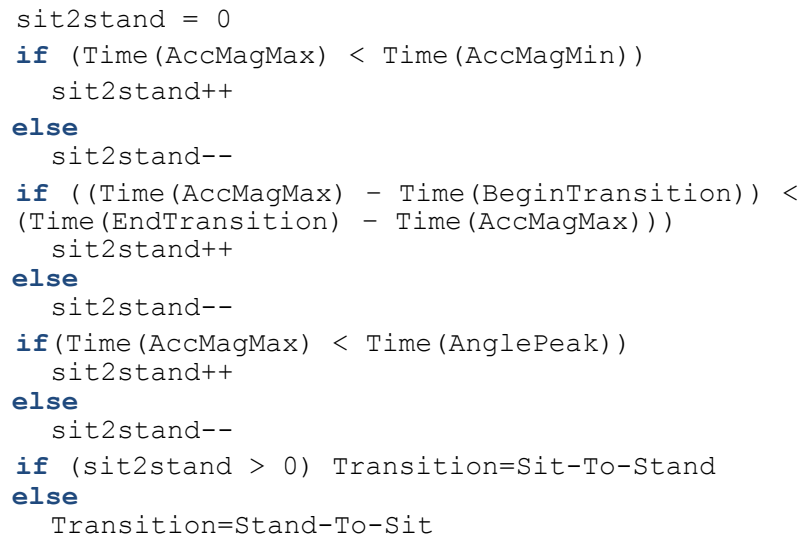

Figure 2. Algorithm for determining type of posture transition.

The next step is to determine the type of the posture transition. In order to distinguish between the sit-to-stand and the stand-to-sit transitions, we employ multiple criteria that consider ordering of the characteristic points in time. The type of the transition is determined by a voting system described in Figure 2.

\section{B. Dynamic Heart Response}

Heart activity is continually recorded. In addition, dynamic heart response to a sit-to-stand posture transition is characterized in near real-time. The characterization is performed once the transition is detected and involves determining characteristic points as shown in Figure 1(b): (i) heart rate at the beginning of posture transition $\left(\mathrm{HR}_{\mathrm{b}}\right)$, (ii) the maximum heart rate after the transition is performed $\left(\mathrm{HR}_{\mathrm{a}}\right)$, and (iii) timestamp of the maximum heart rate. From these parameters we calculate the heart rate difference $\left(\mathrm{HR}_{\mathrm{diff}}\right)$ and the time needed to reach the maximum heart rate from the beginning of the transition $\left(T_{\text {HRMAX }}\right)$. To find $H_{a}$, we search for the maximum heart rate in a time window of 13 seconds after EndTransition. If it is detected at the end of this window, we continue the search for the maximum beyond this point in time, until the heart rate starts decreasing.

For each sit-to-stand transition we record the following parameters: (a) Time(BeginTransition), (b) $\mathrm{T}_{\text {Transition }}=$ Time(EndTransition)-Time(BeginTranstion), (c) $\mathrm{HR}_{\mathrm{a}}, \mathrm{HR}_{\mathrm{b}}$, $\mathrm{HR}_{\text {diff }}$, (d) $\mathrm{T}_{\text {HRMAX }}$, (e) $\mathrm{T}_{\text {SIT }}$, and (f) $\mathrm{T}_{\text {STAND }}$, where $\mathrm{T}_{\text {SIT }}$ is the duration of seating before the transition, and $\mathrm{T}_{\mathrm{STAND}}$ is duration of standing still after the transition. $\mathrm{T}_{\text {SIT }}$ and $\mathrm{T}_{\text {STAND }}$ can help further characterize posture transitions: e.g., in analysis we would consider only transitions for which $\mathrm{T}_{\text {SIT }}$ is longer than a certain time period (e.g., 60 seconds) and $\mathrm{T}_{\text {STAND }}$ is longer than $\mathrm{T}_{\text {HRMAX }}$.

\section{EXPERIMENTAL SETUP}

We record signals during a series of cued posture transitions driven by our custom program [21]. The protocol includes (1) Quite sitting for $5 \mathrm{~min}$, (2) Stand still for $30 \mathrm{sec}$, (3) Quite sitting for $2 \mathrm{~min}$, (4) Stand still for $30 \mathrm{sec}$, (4) Sitting \& working on computer for $1 \mathrm{~min}$, (5) Walking for $30 \mathrm{sec}$, (6) Sitting and working on computer for $1 \mathrm{~min}$, (7) Walking for $30 \mathrm{sec}$, (8) Sitting and working on computer for $1 \mathrm{~min}$, (9) Walking for $30 \mathrm{sec}$, and (10) Sitting and working on computer for $1 \mathrm{~min}$.

In addition, we record videos of experiments using the custom program that captures video and synchronizes with other measurements and the cues. The experiments with multiple participants were conducted in the Real-time Physiological Monitoring Lab [21] and Autonomous Tracking Optical Measurement (ATOM) Lab. The ATOM lab allows accurate tracking of reflective markers using 33 Vicon T40 series IR cameras [22].

Our algorithms for detection and characterization of posture transitions rely exclusively on the data from the Zephyr monitor, and we use the Vicon system only for algorithms verification. The experiments in the ATOM lab allow us to accurately capture body movements during posture transitions and thus precisely determine the beginning and the end of each posture transition. The users wear the Zephyr BioHarness monitor and strategically placed reflective markers. The Vicon system records the absolute position of each marker with millimeter precision every 20 ms. 


\section{RESUlts, Discussion, AND CONCLUSION}

The inertial and heart activity data are collected on 7 healthy participants who performed the protocol described in the previous section. To verify accuracy of posture detection algorithm we compare the transitions reported by the program with actual transitions from the protocol and absolute positions and timing recorded using the Vicon system. We found that the proposed algorithm performs flawlessly recognizing correctly all sit-to-stand and stand-to-sit transitions correctly.

Table 1 shows the average and the standard deviation of a subset of parameters characterizing dynamic heart response to sit-to-stand transitions in the protocol for all subjects. The total averages and standards deviation for all subjects are as follow: $\mathrm{T}_{\text {Transition }}=2.7 \pm 0.69 \mathrm{~s}, \quad \mathrm{HR}_{\text {diff }}=27.36 \pm 9.30 \mathrm{bpm}$, $\mathrm{HR}_{\mathrm{b}}=63.30 \pm 9.02 \mathrm{bpm}, \mathrm{HR}_{\mathrm{a}}=90.66 \pm 10.09 \mathrm{bpm}$. The results show a significant change of heart rate $\left(\mathrm{HR}_{\mathrm{diff}}\right)$ for each subject indicating potential of the proposed parameters in characterization sit-to-stand transition.

Table 1. The average and the standard deviation of a subset of parameters characterizing dynamic heart response to sit-to-stand transitions for 7 subjects (S1-S7).

\begin{tabular}{|c|c|c|c|c|}
\hline Subject & $\mathrm{T}_{\text {Transition }}[\mathrm{s}]$ & $\mathrm{HR}_{\mathrm{b}}[\mathrm{bpm}]$ & $\mathrm{HR}_{\text {diff }}[\mathrm{bpm}]$ & $\mathrm{T}_{\text {HRMAX }}[\mathrm{s}]$ \\
\hline $\mathrm{S} 1$ & $2.97 \pm 0.46$ & $66.25 \pm 5.36$ & $36.75 \pm 5.01$ & $11.90 \pm 2.39$ \\
\hline $\mathrm{S} 2$ & $2.73 \pm 0.63$ & $81.2 \pm 4.82$ & $18.79 \pm 4.41$ & $8.99 \pm 2.08$ \\
\hline $\mathrm{S} 3$ & $2.32 \pm 0.48$ & $59.18 \pm 5.55$ & $37.23 \pm 5.44$ & $13.64 \pm 2.46$ \\
\hline S4 & $1.99 \pm 0.74$ & $53.66 \pm 4.23$ & $32.73 \pm 6.33$ & $12.16 \pm 3.62$ \\
\hline S5 & $2.57 \pm 0.43$ & $58.78 \pm 0.69$ & $25.00 \pm 4.48$ & $13.05 \pm 2.49$ \\
\hline S6 & $2.74 \pm 0.47$ & $61.12 \pm 2.53$ & $26.51 \pm 3.53$ & $9.22 \pm 4.58$ \\
\hline S7 & $3.61 \pm 0.50$ & $62.90 \pm 1.73$ & $14.48 \pm 3.66$ & $14.01 \pm 2.58$ \\
\hline
\end{tabular}

This paper's contributions are as follows. First, we developed algorithms that reliably detect posture transitions by processing data from a single $3 \mathrm{D}$ accelerometer built-in in a commercially available physiological monitor. Next, we proposed parameters for characterization of dynamic heart response to sit-to-stand transitions, and developed algorithms for their extraction. Finally, we implemented and successfully tested the imWell system on seven participants during structured experiments.

Whereas the goal of this paper was to develop the enabling technology for unobtrusive capturing of heart dynamic response to posture transitions, further research is needed to develop methods to assess cardiac status and fitness using the proposed parameter and to provide feedback and guidance to the users.

\section{ACKNOWLEDGMENT}

We are grateful to Dr. Nathan Slegers, Director of the ATOM Lab at UAHuntsville for help with the experiments with the Vicon system and Dr. Bruce Johnson from Mayo Clinic and Dr. Robert Lindquist from UAHuntsville for discussions and suggestions.

\section{REFERENCES}

[1] "Zephyr Technology Corporation," Zephyr Technology Corporation. [Online]. Available: http://www.zephyr-technology.com/. [Accessed: 18Jan-2013]
[2]"Polar Heart Rate Monitors and GPS Sport Watches.” [Online]. Available: http://www.polar.com/en. [Accessed: 18-Jan-2013].

[3] "Quantified Self." [Online]. Available: http://quantifiedself.com/. [Accessed: 18-Jan-2013].

[4] S. Bosch, M. Marin-Perianu, R. Marin-Perianu, P. Havinga, and H. Hermens, "Keep on Moving! Activity Monitoring and Stimulation Using Wireless Sensor Networks," in Smart Sensing and Context, P. Barnaghi, K. Moessner, M. Presser, and S. Meissner, Eds. Springer Berlin Heidelberg, 2009, pp. 11-23.

[5] F. Pitta, T. Troosters, V. S. Probst, M. A. Spruit, M. Decramer, and R. Gosselink, "Quantifying physical activity in daily life with questionnaires and motion sensors in COPD," Eur. Respir. J., vol. 27, no. 5, pp. 10401055, May 2006.

[6] R. Ganea, A. Paraschiv-Ionescu, C. Büla, S. Rochat, and K. Aminian, "Multi-parametric evaluation of sit-to-stand and stand-to-sit transitions in elderly people," Medical Engineering \& Physics, vol. 33, no. 9, pp. 10861093, Nov. 2011

[7] A. Al-Jawad, M. R. Adame, M. Romanovas, M. Hobert, W. Maetzler, M. Traechtler, K. Moeller, and Y. Manoli, "Using multi-dimensional dynamic time warping for TUG test instrumentation with inertial sensors," in 2012 IEEE Conference on Multisensor Fusion and Integration for Intelligent Systems (MFI), 2012, pp. $212-218$.

[8] A. Zijlstra, M. Mancini, U. Lindemann, L. Chiari, and W. Zijlstra, "Sitstand and stand-sit transitions in older adults and patients with Parkinson's disease: event detection based on motion sensors versus force plates," Journal of NeuroEngineering and Rehabilitation, vol. 9, no. 1, p. 75, Oct. 2012.

[9] K. Wongpatikaseree, A. O. Lim, Y. Tan, and H. Kanai, "Range-based algorithm for posture classification and fall-down detection in smart homecare system," in 2012 IEEE 1st Global Conference on Consumer Electronics (GCCE), 2012, pp. $243-247$.

[10] C. Tacconi, S. Mellone, and L. Chiari, "Smartphone-based applications for investigating falls and mobility," in 2011 5th International Conference on Pervasive Computing Technologies for Healthcare (PervasiveHealth), 2011, pp. $258-261$.

[11] B. K. Santhiranayagam, D. T. H. Lai, C. Jiang, A. Shilton, and R. Begg, "Automatic detection of different walking conditions using inertial sensor data," in The 2012 International Joint Conference on Neural Networks (IJCNN), 2012, pp. 1-6.

[12] L. Bao and S. S. Intille, "Activity recognition from user-annotated acceleration data," 2004, pp. 1-17.

[13] H. Ghasemzadeh, V. Loseu, and R. Jafari, "Structural Action Recognition in Body Sensor Networks: Distributed Classification Based on String Matching," IEEE Transactions on Information Technology in Biomedicine, vol. 14, no. 2, pp. 425 -435, Mar. 2010.

[14] J. Lester, T. Choudhury, and G. Borriello, "A Practical Approach to Recognizing Physical Activities," in In Proc. of Pervasive, 2006, pp. 1-16. [15] B. Najafi and K. Aminian, "Body movement monitoring system and method," U.S. Patent EP119513910-Apr-2002.

[16] B. Florentino-Liano, N. O'Mahony, and A. Artes-Rodriguez, "Human activity recognition using inertial sensors with invariance to sensor orientation," in $20123 \mathrm{rd}$ International Workshop on Cognitive Information Processing (CIP), 2012, pp. 1-6.

[17] D. Fuentes, L. Gonzalez-Abril, C. Angulo, and J. A. Ortega, "Online motion recognition using an accelerometer in a mobile device," Expert Systems with Applications, vol. 39, no. 3, pp. 2461-2465, Feb. 2012. [18] A. Godfrey, A. K. Bourke, G. M. Ólaighin, P. van de Ven, and J. Nelson, "Activity classification using a single chest mounted tri-axial accelerometer," Medical Engineering \& Physics, vol. 33, no. 9, pp. 11271135, Nov. 2011

[19] A. Fleury, N. Noury, and M. Vacher, "A Wavelet-Based Pattern Recognition Algorithm to Classify Postural Transitions in Humans," in EUSIPCO 2009, 17th European Signal Processing Conference, 2009. [20] "UAHuntsville mHealth Portal." [Online]. Available: http://portal.mhealth.uah.edu/public/index.php. [Accessed: 04-Feb-2013] [21] "Real-time Physiological Monitoring Laboratory." [Online] Available: http://www.ece.uah.edu/ jovanov/labs/RTPLab/. [Accessed: 24Mar-2012]

[22] “ATOM Lab." [Online]. Available: http://www.eng.uah.edu/ slegers/ATOM.html. [Accessed: 04-Feb-2013]. 